\title{
Analysis of Anti-Xa Concentrations in Patients on Treatment Dose Enoxaparin: A Retrospective Chart Review
}

\author{
Lauren Levine ${ }^{1}$, Nicole Pallme ${ }^{1,}$, Erik Angelotti ${ }^{1}$, Dane Shiltz ${ }^{2, *}$ \\ ${ }^{1}$ Butler University College of Pharmacy and Health Sciences, Indianapolis, 46208, Indiana, United States \\ ${ }^{2}$ Indiana University Health Methodist Hospital, Indianapolis, 46202, Indiana, United States \\ *Corresponding Author: dshiltz@butler.edu
}

Copyright (C) 2013 Horizon Research Publishing All rights reserved.

\begin{abstract}
The purpose of this retrospective chart review was to determine the correlation between full weight-based enoxaparin use and the frequency of anti-Xa concentrations within the defined therapeutic range; to ascertain if anti-Xa monitoring is being appropriately ordered in relation to the timing of enoxaparin dose (after 3 consistent therapeutic doses and 3-5 hours post-dose); to establish if the evidence-based recommended dose adjustment protocol that was studied in the pediatric population was utilized; and if this yielded anti-Xa concentrations within the target range $(0.6-1.1 \mathrm{IU} / \mathrm{mL})$ for an adult population. The data may suggest a lack of correlation between BMI and whether or not the anti-Xa concentration is within the therapeutic range. Further prospective studies are needed to confirm this finding, and to determine the utility of the available dose adjustment nomogram.
\end{abstract}

Keywords Enoxaparin, Anti-Xa, Anticoagulation

\section{Introduction}

Enoxaparin is a low-molecular-weight heparin (LMWH) utilized for deep vein thrombosis (DVT) and pulmonary embolism (PE) prophylaxis and treatment, unstable angina, and acute myocardial infarction. Antithrombotic activity is achieved by binding to antithrombin III (AT III), which inhibits coagulation factors Xa and thrombin (IIa). There is more selectivity for inhibition of factor Xa over IIa, in a 2.7-4:1 ratio. As a result, anti-factor Xa activity can be utilized to determine the degree of anticoagulation in patients receiving treatment dose enoxaparin.[1]

The 2012 CHEST guidelines recommend a therapeutic dose of $1 \mathrm{mg} / \mathrm{kg}$ subcutaneously (SC) every 12 hours or 1.5 $\mathrm{mg} / \mathrm{kg} \mathrm{SC}$ every 24 hours. The dose is adjusted for patients with a creatinine clearance $(\mathrm{CrCl}) \leq 30 \mathrm{~mL} / \mathrm{min}$ to $1 \mathrm{mg} / \mathrm{kg}$ every 24 hours. Due to the lack of data in patients with a $\mathrm{CrCl}<10 \mathrm{~mL} / \mathrm{min}$ and those on dialysis, enoxaparin is generally not recommended in these patients. Therapeutic enoxaparin dosing should be based on actual body weight.[2]

Enoxaparin has more predictable pharmacokinetics than unfractionated heparin.[3] As a result, routine monitoring is not recommended. However, in specific patient populations anti-Xa monitoring can be utilized to determine the degree of anticoagulation.[2] These patient populations include morbid obesity $\left(\mathrm{BMI} \geq 40 \mathrm{~kg} / \mathrm{m}^{2}\right)$, renal impairment $(\mathrm{CrCl} \leq$ $30 \mathrm{~mL} / \mathrm{min}$ ), and pregnancy.[4] Data is lacking in morbidly obese patients because many studies exclude this patient population. Many physicians are reluctant to utilize the full weight-based dose due to the concern for over anticoagulation. $[5,6]$

On average, anti-Xa concentrations peak 4 hours post-dose, but can occur between 3 to 5 hours post-dose in healthy individuals. The peak provides the best indication for enoxaparin anticoagulant activity.[2] Steady state concentration is reached on day 2, after two doses of 1.5 $\mathrm{mg} / \mathrm{kg}$ every 24 hours or three doses of $1 \mathrm{mg} / \mathrm{kg}$ every 12 hours.[1] As a result, anti-Xa concentrations should be drawn 4 hours post-dose on day 2. The therapeutic range may differ based on the laboratory analyzing the specimen. In general, the therapeutic range is $0.6-1 \mathrm{IU} / \mathrm{mL}$ for the 1 $\mathrm{mg} / \mathrm{kg}$ every 12 hour dosing or $1-2 \mathrm{IU} / \mathrm{mL}$ for the $1.5 \mathrm{mg} / \mathrm{kg}$ every 24 hour dosing.[2,7] The Indiana University Health laboratory where this study was conducted utilized 0.6-1.1 $\mathrm{IU} / \mathrm{mL}$ as their therapeutic range.

Once an anti-Xa concentration is obtained, there is limited available data on how to adjust the dose. Monagle and colleagues present a dosing nomogram for pediatric patients (see Table 1).[8] However, there are no available dose adjustment nomograms in adults. This pediatric nomogram has not previously been evaluated in adults. 
Table 1. Pediatric Dosing Nomogram ${ }^{8}$

\begin{tabular}{cccc}
\hline Anti-Factor Xa Level IU/mL & Hold Next Dose? & Dose Change? & Repeat Anti-Factor Xa \\
\hline$<0.35$ & No & Increase by $25 \%$ & $4 \mathrm{~h}$ after next dose \\
$0.35-0.49$ & No & Increase by $10 \%$ & $4 \mathrm{~h}$ after next dose \\
$0.5-1.0$ & No & No & Next day \\
$1.1-1.5$ & No & Decrease by $20 \%$ & Before next dose \\
$1.6-2.0$ & $3 \mathrm{~h}$ & Decrease by $30 \%$ & $\begin{array}{c}\text { Before next dose then } 4 \mathrm{~h} \text { after } \\
\text { next dose }\end{array}$ \\
& Until anti-factor Xa $0.5 \mathrm{IU} / \mathrm{mL}$ & Decrease by $40 \%$ & $\begin{array}{c}\text { Before next dose, if not }<0.5 \\
\text { IU } / \mathrm{mL}, \text { repeat q12h }\end{array}$ \\
\hline
\end{tabular}

Adapted from Monagle P, Michelson AD, Bovill E, Andrew M. Chest. 2001; 119 (1 Suppl): 344S-370S

Bazinet and colleagues[9] conducted a prospective study comparing anti-Xa activity of once-daily vs. twice-daily regimens, non-obese patients (BMI $18-30 \mathrm{~kg} / \mathrm{m}^{2}$ ) vs. obese patients (BMI $\left.>30 \mathrm{~kg} / \mathrm{m}^{2}\right)$, and renal impairment vs. normal renal function. Dosing was consistent with the 2012 CHEST guideline recommendations. Their data suggested that once-daily and twice-daily regimens produce similar anti-Xa levels. Anti-Xa concentrations were also similar in obese and non-obese patients. Patient weight ranged from 40-159 kg. As a result, they concluded that no dosage adjustment was necessary for patients weighing up to $159 \mathrm{~kg}$.[9] However, data is lacking in patients weighing greater than $159 \mathrm{~kg}$. Further research is needed to evaluate anti-Xa concentrations following therapeutic weight-based dosing in obese and morbidly obese patients, the appropriateness of anti-Xa lab draws in clinical practice, and the possible utility of the pediatric dose adjustment nomogram in the adult population. This retrospective chart review analyzes anti-Xa concentrations after therapeutic enoxaparin treatment and subsequent dose adjustments.

\section{Materials and Methods}

This was a retrospective cohort study. We conducted a Cerner query from May 1, 2011 to April 31, 2012 of patients receiving treatment dose enoxaparin and at least one anti-Xa concentration during the same admission at an Indiana University Health facility (i.e., Methodist, University, North, Arnett, and West). The query yielded 100 patients.

Patients were included if they were at least 18 years of age, had an anti-Xa concentration obtained after at least 3 doses of enoxaparin on a stable therapeutic-dose regimen, had stable renal function, and had an indication for treatment dose enoxaparin. Exclusion criteria included prisoners, non-enoxaparin anticoagulation that may interfere with anti-Xa concentrations, anti-Xa concentration not obtained at steady state, unstable renal function necessitating change in enoxaparin dose within 3 doses of anti-Xa lab draw, prophylactic dose enoxaparin within 3 doses of anti-Xa lab draw, treatment dose of enoxaparin not based on actual body weight, hemodialysis, peritoneal dialysis, continuous renal replacement therapy, estimated creatinine clearance $<10$ $\mathrm{mL} / \mathrm{min}$, additional or missed enoxaparin doses within 3 doses of anti-Xa lab draw, or outpatient treatment.

\subsection{Objectives}

The primary study objectives were to determine the correlation between full weight-based enoxaparin use and the frequency of anti-Xa concentrations within the defined therapeutic target range $(0.6-1.1 \mathrm{IU} / \mathrm{mL})$, to determine if anti-Xa monitoring is being appropriately ordered in relation to the timing of the enoxaparin dose, to determine if evidence-based recommended dose adjustment protocols are utilized, and to determine if there is a difference upon repeat anti-Xa concentration between the cohort whose prescribers followed the pediatric dosing protocol and those prescribers who did not with regard to establishing an anti-Xa concentration within the recommended target range.

Table 2. Baseline Demographics

\begin{tabular}{ccc}
\hline & Median & $\begin{array}{c}\text { Interquartile Range } \\
(\mathrm{IQR})\end{array}$ \\
\hline Age (years) & 58 & 27.3 \\
ABW (kg) & 97.5 & 37.5 \\
IBW (kg) & 67 & 19.0 \\
BMI (kg/m2) & 34 & 14.8 \\
LOS (days) & 10.5 & 25.3 \\
Max SCr (mL/min) & 1.09 & 0.45 \\
Min CrCl (mL/min) & 65.5 & 49.8 \\
Min SCr (mL/min) & 0.83 & 0.51 \\
Max CrCl (mL/min) & 111.5 & 75.0 \\
\hline
\end{tabular}

\subsection{Statistical Analysis}

Twenty-two out of one-hundred patients identified in the Cerner query were included. Due to the non-normal distribution of data, descriptive statistics are used to present the data, including median and interquartile ranges. As a result of the small patient population and lack of power, p-values were not able to be utilized.

\subsection{Baseline Demographics}


Baseline demographics are presented in Table 2. Eleven $(50 \%)$ of the twenty-two patients were male. The indications for treatment dose enoxaparin included seventeen patients with venous thromboembolism (VTE), two patients with atrial fibrillation, three patients with active malignancies, and two patients with thrombophilia and previous occurrences of VTE.

Table 3. Results-Stratified by $1^{\text {st }}$ Anti-Xa

\begin{tabular}{|c|c|c|c|}
\hline \multicolumn{4}{|c|}{ Concentration } \\
\hline Variable & $\begin{array}{l}\text { Anti-Xa in } \\
\text { Range } \\
(\mathbf{N}=17)\end{array}$ & $\begin{array}{c}\text { Anti-Xa Out } \\
\text { of Range } \\
(\mathrm{N}=5)\end{array}$ & P-Value \\
\hline $\begin{array}{l}\text { Enoxaparin dose } \\
\text { (mg) }\end{array}$ & $\begin{array}{c}100 \\
(60,180)\end{array}$ & $\begin{array}{c}120 \\
(80,140)\end{array}$ & 0.45 \\
\hline $\begin{array}{c}\text { Enoxaparin dose } \\
\text { (mg/kg/dose) }\end{array}$ & $\begin{array}{c}1.01 \\
(0.83,1.41)\end{array}$ & $\begin{array}{c}1.03 \\
(0.98,1.41)\end{array}$ & 0.62 \\
\hline $\begin{array}{l}\# \text { doses prior to } \\
1^{\text {st }} \text { anti-Xa }\end{array}$ & $\begin{array}{c}4 \\
(3,18)\end{array}$ & $\begin{array}{c}4 \\
(3,25)\end{array}$ & 0.69 \\
\hline BMI $\left(\mathrm{kg} / \mathrm{m}^{2}\right)$ & $\begin{array}{c}35.5 \\
(18,68)\end{array}$ & $\begin{array}{c}27 \\
(26,45)\end{array}$ & 0.65 \\
\hline $\begin{array}{c}\text { Time (hrs) of } \\
\text { anti-Xa after last } \\
\text { dose }\end{array}$ & $\begin{array}{c}4 \\
(3,5)\end{array}$ & $\begin{array}{c}4 \\
(3,4)\end{array}$ & 0.51 \\
\hline
\end{tabular}

Note: Medians presented with minimum, maximum values indicated in parentheses.

\section{Results}

The overall median enoxaparin weight-based dose was $1.025 \mathrm{mg} / \mathrm{kg} / \mathrm{dose}$ (IQR 0.078). Twenty patients received every twelve hour dosing, and two patients received every twenty-four hour dosing. The overall median first anti-Xa concentration was $0.83 \mathrm{IU} / \mathrm{mL}$ (see Table 3 ).

Five anti-Xa concentrations were outside of the therapeutic range, and seventeen anti-Xa concentrations were within the therapeutic range. The BMI ranges for patients in the within-range and out-of-range groups were $18-68 \mathrm{~kg} / \mathrm{m}^{2}$ and $26-45 \mathrm{~kg} / \mathrm{m}^{2}$ respectively (see Figure 1).

Only two of the five enoxaparin doses resulting in an out-of-range anti-Xa concentration were adjusted (see Table 4). These two out-of-range anti-Xa levels were $1.27 \mathrm{IU} / \mathrm{mL}$ and $1.26 \mathrm{IU} / \mathrm{mL}$. According to the dose adjustment nomogram presented by Monagle and colleagues[8], it is recommended to decrease the enoxaparin dose in both cases by $20 \%$. In both cases, the dose was adjusted based on the literature. However, a second anti-Xa concentration was drawn in only one of these cases. Despite the $20 \%$ decrease in enoxaparin dose, the anti-Xa concentration increased from 1.26 to 1.4. Upon further investigation this patient's serum creatinine increased from $0.7 \mathrm{mg} / \mathrm{dL}$ to $1.4 \mathrm{mg} / \mathrm{dL}$, explaining the increase in the second anti-Xa concentration.

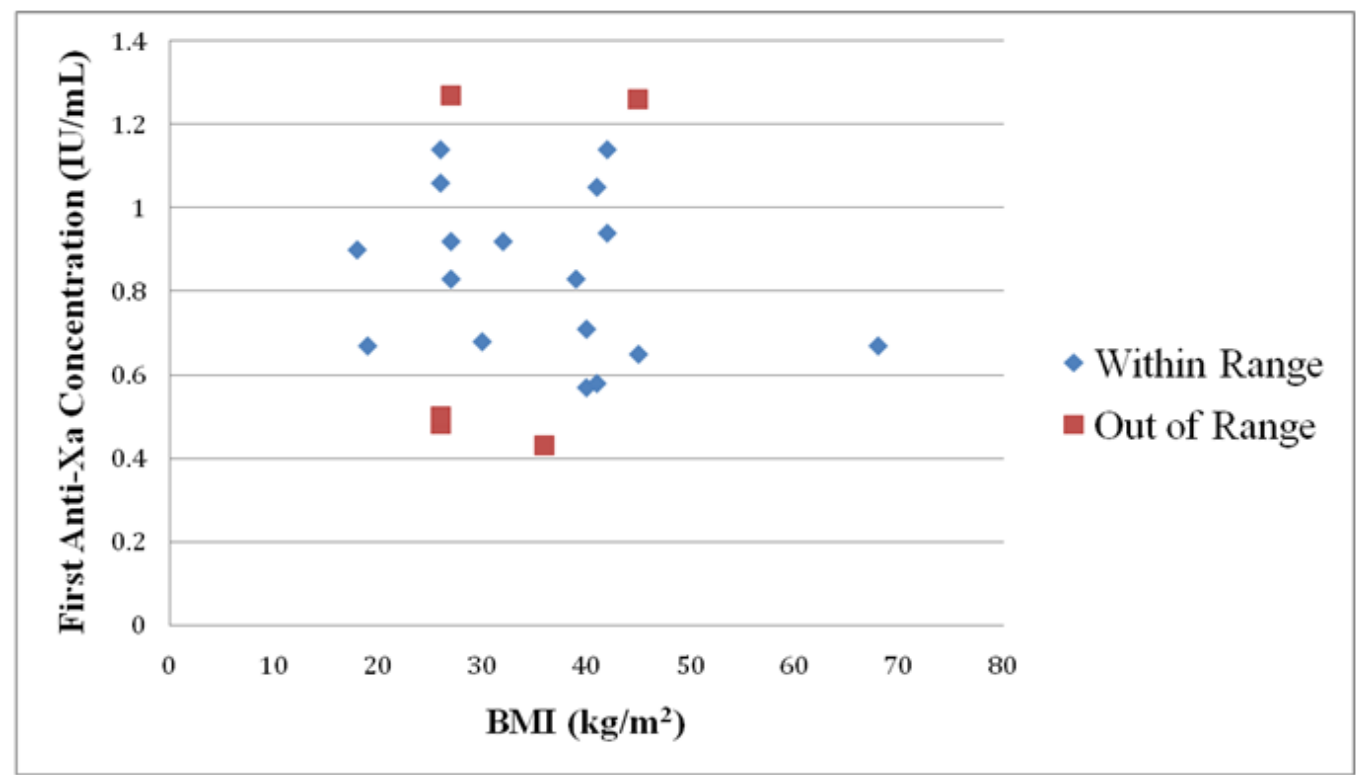

Note: the therapeutic range of the IU Health lab is 0.6 to $1.1 \mathrm{IU} / \mathrm{mL}$, and the level obtained is rounded to the nearest tenth

Figure 1. First anti-Xa concentrations classified as "within range" or "out of range" based on each patient's BMI

Table 4: Doses Adjusted After First Anti-Xa Concentration

\begin{tabular}{ccccccccc}
\hline Dose $(\mathrm{mg})$ & $\begin{array}{c}\text { Dose } \\
(\mathrm{mg} / \mathrm{kg})\end{array}$ & $\begin{array}{c}\text { Interval } \\
(\mathrm{hrs})\end{array}$ & $\begin{array}{c}\text { First Anti-Xa } \\
(\mathrm{IU} / \mathrm{mL})\end{array}$ & $\begin{array}{c}\text { New dose } \\
(\mathrm{mg})\end{array}$ & $\begin{array}{c}\text { New dose } \\
(\mathrm{mg} / \mathrm{kg})\end{array}$ & $\begin{array}{c}\text { Interval } \\
(\mathrm{hrs})\end{array}$ & $\begin{array}{c}\text { Second } \\
\text { Anti-Xa } \\
(\mathrm{IU} / \mathrm{mL})\end{array}$ & $\begin{array}{c}\text { Dose change with } \\
\text { literature? }\end{array}$ \\
\hline 120 & 1.41 & $\mathrm{q} 12 \mathrm{~h}$ & 1.27 & 100 & 1.18 & $\mathrm{q} 12 \mathrm{~h}$ & $\mathrm{~N} / \mathrm{A}$ & Yes \\
160 & 1.03 & $\mathrm{q} 12 \mathrm{~h}$ & 1.26 & 130 & 0.84 & $\mathrm{q} 12 \mathrm{~h}$ & 1.4 & Yes \\
\hline
\end{tabular}


Table 5. Exclusions

\begin{tabular}{cc}
\hline Exclusion Criteria & Number of Exclusions \\
\hline Prisoners & 0 \\
Active pregnancy & 0 \\
Persons $<18$ years old & 0 \\
Allergy to heparin products & 0 \\
regimen & 35 \\
Anti-Xa lab obtained before patient received $\geq 3$ consecutive treatment doses of a stable enoxaparin & 31 \\
Anti-Xa lab obtained $<3$ or $>5$ hours after enoxaparin dose & 30 \\
Not receiving a treatment dose of enoxaparin equivalent to ABW & 3 \\
Patient receiving dialysis or CrCl $<10 \mathrm{~mL} /$ min & 6 \\
No anti-Xa levels drawn & 11 \\
\hline Tnstable renal function necessitating a dosage adjustment within 3 doses of anti-Xa level & 1 \\
TOTAL & 117 \\
\hline
\end{tabular}

\subsection{Exclusions}

In order to determine the appropriateness of anti-Xa lab draws in clinical practice, reasons for exclusion were analyzed. Seventy-eight patients were excluded for 117 reasons. Patients could be represented in more than one exclusion category. Sixty-six of the 117 reasons for exclusion $(56 \%)$ were inappropriate lab draws. These included anti-Xa concentrations obtained outside of the 3-5 hour post-dose window or prior to steady state. All reasons for exclusion are presented in Table 5 .

\subsection{Limitations}

The retrospective nature of the study has inherent limitations. We must rely on the accuracy of the charts that we reviewed and were limited by the available data. As a result of the lack of control over which dose each patient received and when the anti-Xa concentration was drawn, many patients were excluded. A small patient population remained resulting in an inability to properly analyze the primary objectives. The distribution was non-normal and therefore Mann-Whitney tests were used to calculate medians, IQR's, and minimum and maximum values. Each calculated $p$-value did not demonstrate statistical significance (see Table 3). Only two patients had dose adjustments, and only one patient had a repeat anti-Xa level drawn after dose adjustment. As a result, we were unable to analyze the utility of the dosing nomogram presented by Monagle and colleagues in the adult population.[8]

Lastly, we did not analyze bleeding and thrombotic events to determine a correlation between the target anti-Xa range $(0.6-1.1 \mathrm{IU} / \mathrm{mL})$ and adequate anticoagulation. A previous study by Spinler and colleagues determined that weight-based enoxaparin dosing in obese patients with non-ST segment elevation myocardial infarction reduced clinical endpoints without a significant risk of major hemorrhage.[10] Another study by Barba and colleagues found that patients weighing over $100 \mathrm{~kg}$ and those weighing between $50-100 \mathrm{~kg}$ on enoxaparin therapy had similar incidences of recurrent VTE, fatal pulmonary embolism, and major bleeding complications.[11] However, further studies are needed to assess target anti-Xa levels and clinical outcomes together.

\section{Discussion}

While our study was underpowered to determine statistical significance, the data may suggest that there is no correlation between BMI and whether or not the anti-Xa concentration is within the target range (see Figure $1 \&$ Table 3). These results are similar to those of the study conducted by Bazinet and colleagues. They determined that there was no statistically significant difference between anti-Xa levels drawn in obese and non-obese study groups after treatment-dose enoxaparin. They included weights up to 159 $\mathrm{kg}$, and thus determined that no dosage adjustment was necessary up to $159 \mathrm{~kg}$.[9] Our study produced similar results with patients weighing up to $191 \mathrm{~kg}$.

Based on the patients excluded from our study, it is apparent that anti-Xa concentrations are often drawn inappropriately in clinical practice. It is important to properly educate health care prescribers and nurses on correctly ordering and drawing anti-Xa levels, respectively. The patient should be on a consistent regimen for at least 3 doses, and the level should be drawn between 3 and 5 hours post-dose. This laboratory test is expensive, and it is important to be able to utilize the result to guide dosage adjustment.

Of the two doses that were adjusted following the first anti-Xa concentration, both were consistent with the currently available evidence-based recommendations. Literature recommendations, however, are only available for the pediatric population. As a result, it is important to determine if these recommendations can be extrapolated to the adult population. Our study did not include enough patients with dose adjustments to analyze the use of this 
pediatric nomogram in adults. We were also unable to draw any conclusions from the data about repeat anti-Xa levels after dose adjustment because only one patient had a second level drawn.

\section{Conclusion}

The data from this retrospective chart review may suggest that a correlation is lacking between BMI and whether or not the anti-Xa concentration is within the therapeutic range. However, higher powered studies would be needed to verify this result. Further prospective studies would be beneficial to determine the utility of the currently available pediatric dose adjustment nomogram in adults.

\section{REFERENCES}

[1] Lovenox [package insert]. Bridgewater, NJ: Sanofi-Aventis; 2011.

[2] Garcia DA, Baglin TP, Weitz JI, Samama MM. Parenteral anticoagulants: Antithrombotic therapy and prevention of thrombosis, 9th ed: American College of Chest Physicians evidence-based clinical practice guidelines. Chest. 2012; 141(2)(Suppl): e24S-e43S.

[3] Reis Macedo LG, Oliveira L, Pintao MC, Garcia AA, Pazin-Filbo A. Error in body weight estimation leads to inadequate parenteral anticoagulation. Am J Emerg Med. 2011; 29(6): 613-17.

[4] Sanderink GJ, Liboux AL, Jariwala N, et al. The pharmacokinetic and pharmacodynamics of enoxaparin in obese volunteers. Clin Pharmacol Ther. 2002; 72: 318-18.

[5] Nutescu EA, Spinler SA, Wittkowsky A, Dager WE. Low-molecular-weight heparins in renal impairment and obesity: available evidence and clinical practice recommendations across medical and surgical settings. Ann Pharmacother. 2009; 43: 1064-83.

[6] Spinler SA, Ou FS, Roe MT, et al. Weight-based dosing of enoxaparin in obese patients with non-ST-segment elevation acute coronary syndromes: results from the CRUSADE initiative. Pharmacotherapy. 2009; 29(6): 631-638.

[7] Laposata M, Green D, Van Cott EM, Barrowcliffe TW, Goodnight SH, Sosolik RC. College of American Pathologists Conference XXXI on laboratory monitoring of anticoagulant therapy: the clinical use and laboratory monitoring of low-molecular-weight heparin, danaparoid, hirudin and related compounds, and argatroban. Arch Pathol Lab Med. 1998; 122: 799-807.

[8] Monagle P, Michelson AD, Bovill E, Andrew $\mathrm{M}$. Antithrombotic therapy in children. Chest. 2001; 119(1 Suppl): 344S-370S.

[9] Bazinet A, Almanric K, Brunet C, et al. Dosage of enoxaparin among obese and renal impairment patients. Thromb Res. 2005; 116: 41-50.

[10] Spinler SA, Inverso SM, Cohen M, Goodman SG, Stringer KA, Antman EM. Safety and efficacy of unfractionated heparin versus enoxaparin in patients who are obese and patients with severe renal impairment: analysis from the ESSENCE and TIMI 11B studies. Am Heart J. 2003; 146: 33-41.

[11] Barba R, Marco J, Martin-Alvarez H, Rondon P, Fernandex-Capitan C, Garcia-Bragado F, Montreal M. The influence of extreme body weight on clinical outcome of patients with venous thromboembolism: findings from a prospective registry (RIETE). J Thromb Haemost. 2005; 3 : 856-62. 